\title{
Is high-power radiofrequency ablation of atrial fibrillation less painful than standard approach in conscious patients?
}

\author{
Maciej Wójcik ${ }^{1}$, Przemysław Zając ${ }^{1}$, Radosław Gęca ${ }^{2}$, Paweł Błaszkiewicz ${ }^{2}$, Jan Zarębski ${ }^{2}$, \\ Andrzej Wysokinski ${ }^{1}$, and Elżbieta Czekajska-Chehab ${ }^{1}$ \\ ${ }^{1}$ Medical University of Lublin \\ ${ }^{2}$ Samodzielny Publiczny Szpital Kliniczny nr 4 w Lubline
}

August 6, 2020

\begin{abstract}
Background: High-power radiofrequency ablation (HPRFA) appears to be a novel concept for atrial fibrillation (AF) treatment but there are scarce data in conscious patients. The lesion size index (LSI) value has been associated with durability of pulmonary vein isolation (PVI) lesions. Objectives: We hypothesised that HPRFA applications based on LSI were not inferior to standard approach in terms of patient's pain sensationas well as safety, effectiveness, and procedure duration. Methods: Retrospectively, we analysed 218 patients who had performed ablation of AF based on LSI (LSI 4-4.5 on posterior wall, LSI 5-5.5 in other locations) who were propensity score matched to 3 different RF power settings: group- $30 \mathrm{~W}$ (45oC, 30 W and $25 \mathrm{~W}$ on posterior wall), group- $40 \mathrm{~W}(45 \mathrm{oC}, 40 \mathrm{~W})$ and group- $50 \mathrm{~W}(45 \mathrm{oC}, 50 \mathrm{~W})$. Results: Comparing group- $30 \mathrm{~W}$ vs group40W and group-50W, procedure and left atrium dwell time (minutes) were 190 $\pm 32,161 \pm 41,102 \pm 8(\mathrm{p}<0.0001)$ and $154 \pm 53$, $113 \pm 29,79 \pm 10(\mathrm{p}<0.0001)$, respectively. With comparable number of RF applications $(\mathrm{p}>0.05)$ between the groups, total $\mathrm{RF}$ and fluoroscopy time (minutes) were $62 \pm 17,38 \pm 10,28 \pm 5(\mathrm{p}<0.0001)$ and $6 \pm 3,6 \pm 2,4 \pm 1(\mathrm{p}=0.08)$, respectively. Number of stopped painful RF applications were $19 \pm 9,9 \pm 5$ and $7 \pm 2(\mathrm{p}<0.0001)$, respectively. No serious complications were observed in any of the group. No difference in 1-year ablation efficacy was observed between the groups $(\mathrm{p}=0.78)$. Conclusions: HPRFA based on LSI is less painful, faster and safe in conscious patients than standard approach with comparable effectiveness in one year follow-up.
\end{abstract}

\section{ABSTRACT:}

Background: High-power radiofrequency ablation (HPRFA) appears to be a novel concept for atrial fibrillation $(\mathrm{AF})$ treatment but there are scarce data in conscious patients. The lesion size index (LSI) value has been associated with durability of pulmonary vein isolation (PVI) lesions.

Objectives: We hypothesised that HPRFA applications based on LSI were not inferior to standard approach in terms of patient's pain sensationas well as safety, effectiveness, and procedure duration.

Methods: Retrospectively, we analysed 218 patients who had performed ablation of AF based on LSI (LSI 44.5 on posterior wall, LSI 5-5.5 in other locations) who were propensity score matched to 3 different RF power settings: group- $30 \mathrm{~W}\left(45^{\circ} \mathrm{C}, 30 \mathrm{~W}\right.$ and $25 \mathrm{~W}$ on posterior wall), group- $40 \mathrm{~W}\left(45^{\circ} \mathrm{C}, 40 \mathrm{~W}\right)$ and group- $50 \mathrm{~W}$ $\left(45^{\circ} \mathrm{C}, 50 \mathrm{~W}\right)$.

Results: Comparing group-30W vs group-40W and group-50W, procedure and left atrium dwell time (minutes) were $190 \pm 32,161 \pm 41,102 \pm 8(\mathrm{p}<0.0001)$ and $154 \pm 53,113 \pm 29,79 \pm 10(\mathrm{p}<0.0001)$, respectively. With comparable number of RF applications ( $\mathrm{p}>0.05$ ) between the groups, total RF and fluoroscopy time (minutes) were $62 \pm 17,38 \pm 10,28 \pm 5(\mathrm{p}<0.0001)$ and $6 \pm 3,6 \pm 2,4 \pm 1(\mathrm{p}=0.08)$, respectively. Number of stopped painful $\mathrm{RF}$ applications were $19 \pm 9,9 \pm 5$ and $7 \pm 2(\mathrm{p}<0.0001)$, respectively. No serious complications were 
observed in any of the group. No difference in 1-year ablation efficacy was observed between the groups $(\mathrm{p}=0.78)$.

Conclusions: HPRFA based on LSI is less painful, faster and safe in conscious patients than standard approach with comparable effectiveness in one year follow-up.

\section{INTRODUCTION}

Atrial fibrillation (AF) ablation is the most commonly performed radiofrequency (RF) ablation and is usually associated with a long procedural time and pain sensation in conscious patients. Prolonged radiation exposure during the procedure puts the patient and the operator at risk of malignancy and genetic abnormalities. Complications such as asymptomatic cerebral lesions, tamponade, perforation, and also arrhythmia recurrence are associated with longer ablation time.

Radiofrequency application works by an immediate local resistive heating from the high-frequency current passing through the tissue surrounding the catheter tip and a subsequent conductive phase, where the heat dissipation leads to deeper tissue injury [1]. The current density diminishes as the distance from the catheter tip increases which limits the area of resistive heating. A shorter pulse of high-energy RF application at a given contact force has the potential of achieving rapid, more controlled, resistive tissue heating, and avoiding deeper, passive, less controlled heating, which is seen with longer, low-power RF energy applications [1].

Technological advances have opened up the possibility to manipulate lesion characteristics by modulating the power and duration of RF energy [1]. Three key RF application variables: contact force, power and duration are the main factors responsible for a lesion quality $[2,3]$. The commercially available lesion size index (LSI) incorporates these variables and also impedance in one multiparametric factor, that has been validated prospectively resulting in a significant long-term success rate during AF ablation $[4,5]$. The LSI formula was more predictive in terms of lesion formation than each of its components considered alone regarding width and depth in the in vitro model [4].

Given that the average human atrial wall thickness is 2-3 $\mathrm{mm}$ on the smooth surface [6], we hypothesised that the high-power radiofrequency ablation (HPRFA) based on LSI was sufficient to cause thermal injury to this depth, would produce effective transmural lesions, shorten procedure time and, by limiting collateral damage to adjacent structures, make the procedure less painful for a patient.

The goal of this study was to compare three methods of RF ablation based on LSI (4-4.5 on posterior wall and LSI 5-5.5 in other locations) with different power settings: group-30W: standard RF settings of 30 Watts $(\mathrm{W}), 45^{\circ} \mathrm{C}\left(25 \mathrm{~W}\right.$ on posterior wall); group- $40 \mathrm{~W}$ : moderate $\mathrm{RF}$ settings of $40 \mathrm{~W}, 45^{\circ} \mathrm{C}$, and group- $50 \mathrm{~W}$ : high-power RF (HPRF) settings of $50 \mathrm{~W}, 45^{\circ} \mathrm{C}$.

\section{METHODS}

Patients. Retrospectively we analysed 218 patients who had performed ablation of AF and based on inclusion (symptomatic patients with non-valvular AF, aged:18-85, first procedure in left atrium (LA), exclusion of thrombus in LA and left atrial appendage, $\mathrm{EF}>45 \%$ ) and exclusion (age below 18 or above 85 years, $\mathrm{EF}<45 \%$, previous procedure in LA, valvular AF) criteria (Clinical Trial: NCT04250181), we identified 107 patients who were divided into 3 groups (group-30W - standard RF settings, group-40W and group-50W). Finally, after the patients were propensity score matched in a 1:1:1 fashion we identified 30 patients (10 in each group) who were analysed further. The AF type was categorised according to recent definition as paroxysmal (PAF) and persistent (PEAF) [7]. Our study was consistent with the principles summarized in the 1975 Declaration of Helsinki and written informed consent was taken from all participants accordingly (Clinical Trial: NCT04250181). Baseline demographic, clinical and procedural characteristics and follow-up data were recorded for all patients.

Procedure. Each procedure was performed according to a standard protocol, previously described [8]. Briefly, all patients underwent cardio-CT and transesophageal echocardiography (TEE) up to 48 hours prior to the procedure. Antiarrhythmic drugs were stopped at least five half-lives and amiodarone at least 3 months 
before ablation; ss-blockers were allowed according to the protocol. An interrupted anticoagulation approach was used. Proton pump inhibitor was given peri-procedurally. All patients underwent the ablation under conscious "moderate" sedation with intravenous fentanyl only (they remained conscious and responsive to pain throughout the procedure), without the presence of an anaesthesiologist. Vascular access was obtained through a femoral vein. A decapolar catheter was positioned in the coronary sinus. Transseptal puncture was performed using a long steerable sheath (Agilis NxT, Abbott, St. Paul, MN, USA). After accessing the LA, heparin was given intravenously with a target active clotting time (ACT) of 300-350 seconds, and further monitored every 20 minutes. High-density voltage mapping (HDVM) of the LA was performed (EnSite Precision, Abbott, St. Paul, MN, USA; best-duplicate algorithm) during sinus rhythm (or after restoration of sinus rhythm with DC cardioversion) with a circular diagnostic catheter (Advisor FL Sensor Enabled, Abbott, St. Paul, MN, USA). The diagnostic catheter was exchanged with the ablation catheter (TactiCath, Abbott, St. Paul, MN, USA) and antral pulmonary vein isolation (PVI) was performed with desired contact force of 10-20 grams. Left superior and inferior, followed by the right superior and inferior pulmonary veins $(\mathrm{PVs})$, were encircled in pairs on both sides (with additional lines between the ipsilateral veins, if signals were observed after complete PVs encircling) to achieve complete electrical isolation in all PVs.

Additionally, if low voltage area (LVA, defined as 0.1-0.5 mV in bipolar HDVM) [9] was identified it was also targeted and homogenised. The procedure was stopped with confirmation of complete PVI (no capture of atrial tissue observed during stimulation performed from all PVs) and lack of potentials in homogenised LVA.

Follow-up. Prior to discharge from the EP laboratory, all patients underwent transthoracic echocardiography to exclude pericardial effusion. All patients were monitored overnight and oral anticoagulation was introduced the day post-procedure. Additional echocardiography was performed at discharge. All patients were treated with proton pump inhibitors for 6 consecutive weeks. A blanking period of 3 months has been considered [7]. Patients were controlled to be free of antiarrhythmic drugs at the end of the blanking period.

Follow-up visits were scheduled for 1,3,6, 9, and 12 months after the initial procedure for clinical assessment and rhythm monitoring with 7-day Holter ECG recordings at the $3^{\text {rd }}, 6^{\text {th }}$ and $12^{\text {th }}$ month for the first year. Then, bi-annual visits were scheduled with 7-day Holter monitoring once a year. Pacemaker AF data were utilised when available. Patients were advised to obtain an ECG, either at the same institution or elsewhere, when feeling palpitations at times outside of the HolterECG monitoring periods.

The outcome measures were defined as:

Procedure time: total procedure time (needle to needle time).

Left atrial dwelling time: time of LA instrumentation (from transseptal access to removal of a catheter and a sheath).

Voltage mapping time: time needed to perform LA 3D electroanatomical map and HDVM simultaneously.

Total RF time: time of all performed RF applications.

Total number of RF applications: number of all RF applications performed during a procedure.

PVI time: time of isolation of all PVs defined as time from the first to the last RF application needed to reach confirmed PVI.

PVI-RF time: total time of all RF applications needed to reach confirmed isolation of all PVs.

PVI-Number of RF applications: number of all RF applications needed to reach confirmed isolation of all PVs.

PVI-Time of a single RF application: time of a single RF application.

Contact force: mean contact force value reached during RF application. 
Number of stopped RF applications: number of patient's complaints due to pain sensation- surrogate for assessment of the painfulness of the procedure.

X-Ray time: total time of fluoroscopy.

- X-Ray dose: total dose of fluoroscopy.

Complications: both procedure-related complications and all complications in 12 moths follow-up.

Follow-up-30s: absence any arrhythmia recurrence (AR) defined as any documented recurrence of AF and/or atrial tachycardia (AT) and /or atrial flutter (AFL) lasting longer than 30 seconds [10].

Follow-up-24h: absence of any AR defined as any documented recurrence of AF and/or AT and/or AFL lasting longer than 24 hours [11].

\section{STATISTICAL ANALYSIS}

Continuous data are presented as mean +- standard deviation, discrete data as median (interquartile range) and counts (percentage) if categorical. A propensity score was calculated for all eligible patients undergoing first time RF ablation for AF through binary logistic regression with ablation modality (standard vs moderate vs HPRF settings) as the binary outcome and baseline variables were used as covariates for estimating the propensity score. Propensity matching was performed in a 1:1:1 fashion using the nearest neighbour approach with a two decimal calliper for age, sex, body mass index, type of AF, LA size, left ventricular ejection fraction, procedure characteristics (single transseptal puncture with steerable Agilis sheath, performed HDVM of LA during sinus rhythm and RF ablation performed in sedated but fully conscious patients with contact force TactiCath catheter based on LSI settings described above. If no match could be found, then the subject was removed from the analysis. Normal distributed variables were analyzed using paired and unpaired t-test (two-group analysis) and ANOVA (N-group analysis). In the case of non-normal distribution, continuous variables were analyzed using Kruskal-Wallis H test. Kaplan-Meier univariate analysis was used to estimate AR-free survival. All statistical tests were 2 -sided, and $\mathrm{p}<0.05$ was considered significant.

\section{RESULTS}

Patients' characteristics and the results are presented in Table 1 and Table 2, respectively. Briefly:

With the comparable Voltage mapping time of LA, Total number of RF applications and PVI-Number of RF applications, the Procedure time $(\mathrm{p}<0.0001)$, Total RF time $(\mathrm{p}<0.0001)$ and PVI-RF time $(\mathrm{p}<0.0001)$ were significantly shorter in group- $40 \mathrm{~W}$ and group- $50 \mathrm{~W}$ as compared to group-30W. Additional LVA was identified in 6 patients and successfully homogenised.

The acute PVI was reached in all patients. The PVI-Time of a single RF application was significantly shorter in group-40W and group-50W as compared to group-30W $(\mathrm{p}<0.0001)$.

The number of stopped RF applications as a result of pain complained by a patient was significantly lower in group- $40 \mathrm{~W}$ and group- $50 \mathrm{~W}$ as compared to group- $30 \mathrm{~W}(\mathrm{p}=0.01)$, i.e. the procedure was generally less painful for a patient treated with higher RF energy (Figure 1.).

The procedure was comparably safe in all the groups of patients. No major complications, others then related to vascular access (groin hematoma in 2 patients, one pseudoaneurysm without need of surgical intervention), were observed in any of the studied groups. Additionally, shorter LA dwelling time in group$40 \mathrm{~W}$ and group-50W $(\mathrm{p}=0.01)$ decreased the risk of thromboembolic event related to LA instrumentation. No audible pop occurred in any of the groups.

One year follow-up. Asymptomatic AF (8 hours in 7-day Holter ECG) was found at $6^{\text {th }}$ month in a patient treated with $40 \mathrm{~W}$ for PEAF. Another asymptomatic AF (17 hours in 7 day Holter ECG) was identified at the $9^{\text {th }}$ month in a patient treated with $50 \mathrm{~W}$ for PEAF; still the episode was related to vomiting and diarrhoea at that time. Finally, a pacemaker recording showed 4-hour-lasting asymptomatic AF during sleep 
at $9^{\text {th }}$ month in a PAF patient (group-50W) already treated with CPAP for severe nocturnal apnea (Figure 2., Figure 3).

Left atrial AT was documented in 5 patients but was symptomatic AT in 2 PEAF patients (1 patient treated with $30 \mathrm{~W}$ and 1 treated with $50 \mathrm{~W}$ ). Successful AT ablation was performed in both cases. Other 3 patients had documented asymptomatic AT in 7-day Holter ECG monitoring in the $6^{\text {th }}$ month (AT lasting 1 hour related to sudden rise in blood pressure; patient treated with $40 \mathrm{~W}$ for PEAF) and at the $12^{\text {th }}$ month (36 minutes in a patient treated with standard settings for PEAF and 4 hours in a patient treated with $50 \mathrm{~W}$ for PEAF).

Typical cavo-tricuspid AFL was recorded at 9-months follow-up visit in a patient (group-30W) with a history of PAF (only pulmonary vein isolation was performed during the index procedure); he was scheduled for elective AFL ablation and no further AR was observed since.

Total one-year success rate (follow-up-30s and follow-up-24h) was $73 \%$ and $87 \%$, respectively (Figure 2., Figure 3).

1. DISCUSSION

2. MAIN FINDINGS

We utilised commercially available catheters and LSI to perform and compare standard (low-power RF application), moderate and HPRF ablation of AF in conscious patients. Our data show for the first time that in conscious patients HPRF ablation of AF based on LSI was less painful for a patient and faster with the same safety margin and not inferior in outcome as compared to standard RF power approach.

\section{THE PAINFULNESS OF THE PROCEDURE}

While visceral pain is a common finding in patients who undergo catheter ablation in the LA, esophageal warming represents the most probable cause of a patient's symptoms. Pain tends to be higher when the RF is delivered near the esophagus [8]. In sedated patients, pain during ablation is associated with esophageal temperature rise (ETR), and lack of pain is strongly associated with absence of ETR. Pain during RF ablation may thus serve as a predictor of esophageal heating and potential injury [12].

We performed ablation in conscious patients who were able to endure the entire procedure. Our results show that HPRF applications were less painful. As was described previously, duration of each RF delivery was correlated with pain [8]. So shortening the RF application was one of the reason of lower pain intensity. Additionally, most of the damage with HPRF is done during the resistive phase of ablation. Such approach results in shallower lesions which limits collateral damage to adjacent structures and, as a result, reduces the sensation of pain.

In case of longer standard power RF applications (group-30W) the conductive phase of ablation produced prolonged and not fully controlled damage of deeper structures which resulted in more painful procedure in that cohort of conscious patients.

\section{SHORTENING OF PROCEDURE TIME}

The first report of shorter fluoroscopy times and reduction in mean RF times when using the $50 \mathrm{~W}$ approach was published in 2004 [13]. It was confirmed later in 2006 by Nilsson et al. (shorter fluoroscopy times, a significant reduction in RF times and procedure times) [14], by Kanj et al. in 2007 (the $50 \mathrm{~W}$ ablations had shorter fluoroscopy times and atrial instrumentation times) [15] and by Winker et al. in 2011 [16] who observed shorter fluoroscopy and procedure times and reduction in RF time with $50 \mathrm{~W}$ ablation. Recently, Chen et al.reported short procedure and fluoroscopy time in Ablation Indexguided $50 \mathrm{~W}$ ablation [17]. Our results in conscious patients are in line with previously described when general anesthesia was applied [14-17]. We observed significant reduction in procedure time, LA dwell time, RF time, fluoroscopy dose and time. Similarly, Pambrun et al. also observed drastic reduction of procedural duration in the high-power group as a result of shorter time required for lesion creation, more first-pass PVI with fewer acute PVs reconnections 
[18]. The findings were further supported with animal study with novel HPRFA technology (90 W per 4 seconds) by Barkagan et al. [19].

\section{OUTCOME}

The single procedure success rate off antiarrhythmic therapy was comparable to previously reported, despite the fact that we differentiate patients based on RF power used and not type of AF (PAF, PEAF) [10]. Taking into account that only patients with AF lasting longer than 24 hours had an increased risk of stroke or systematic embolism (hazard ratio 3.2), HPRFA seems to be more favourable than standard approach with lower power settings and prolonged RF application time [11].

\section{SAFETY}

All procedures were performed by experienced operator (MW). Our results confirm that high-power $50 \mathrm{~W}$ RF ablation is a safe approach as was reported in large cohort of patients recently [8] as well as during Ablation Index-guided $50 \mathrm{~W}$ ablation [17]. Few authors described severe complications related to $50 \mathrm{~W}$ ablation. Mansour observed cardiac tamponade (5\%) and a cerebrovascular accident (2.5\%) when RF energy was delivered with a maximum temperature of $60^{\circ} \mathrm{C}$ and $50 \mathrm{~W}$ power limited for $12-15$ seconds at each site [13]. Pappone reported two cases of pericardial tamponade, with no thrombo-embolic events or cases of PV stenosis, in 560 patients treated with $100 \mathrm{~W}$ applied for up to 30 seconds [20]. Different techniques and manoeuvres were used by others to lower the complication rates with HPRFA like short duration applications (2-15 seconds) [21] or 12 to 15 seconds at each site, [13] "perpetual motion" of open irrigated tip catheters every 3-10 seconds, [16] the use of a "painting" technique where ablation catheter stayed at each site for 2 to 5 seconds at $50 \mathrm{~W}$ power was moved back and forth until a small area was devoid of electrograms [22] or Ablation Index-guided $50 \mathrm{~W}$ ablation [17]. Recently, the novel HPRFA approach demonstrated in swine model also confirmed its safety profile that was comparable to conventional ablation [19].

Our RF applications were limited by LSI and each application was started only when the desired contact force (10-20 grams) was reached. It resulted in mean 18 and 16 seconds RF application time in group-40W and group-50W, respectively. In few cases where we could not stabilized the catheter to reach contact force $>10$ grams but at least 5 grams were achieved, the $40 \mathrm{~W}$ or $50 \mathrm{~W}$ applications were stopped at 30 seconds even if desired LSI was not reached. Eventually, we did not observed any major complications in any of the studied groups.

\section{STUDY LIMITATIONS}

It was retrospective observational study and performed in a small cohort of patients. There was a small number of individuals in which RF ablation was performed with moderate power settings of $40 \mathrm{~W}$. We promptly changed our protocol to $50 \mathrm{~W}$ RF ablation which limited the number of patients in group- $40 \mathrm{~W}$, and eventually propensity score matching identified 30 patients included in the study.

The threshold for pain is individual for certain patient. Still, the need to cease the RF application seems to be the best clinical surrogate parameter for pain related to possible collateral damage to adjacent structures.

Furthermore, ECG and Holter monitoring were recorded at limited time points. Some episodes of AR could be missed during follow up.

\section{CONCLUSIONS}

To our best knowledge this is the first report which shows that HPRF $(40 / 50 \mathrm{~W})$ ablation of AF based on LSI is less painful, faster and safe procedure for a conscious patients. Its effectiveness in one year follow-up is comparable to standard approach. Prospective randomized trials in a larger cohort of patients are required to confirm the benefits of such strategy.

\section{REFERENCES}

1. Patel PJ, Padanilam BJ. High-power short-duration ablation: Better, safer, and faster? J Cardiovasc Electrophysiol. 2018;29(11):1576-1577. 
2. Nath S, Redick JA, Whayne JG, Haines DE. Ultrastructural observations in the myocardium beyond the region of acute coagulation necrosis following radiofrequency catheter ablation. J Cardiovasc Electrophysiol. 1994;5:838-845.

3. Yokoyama K, NakagawaH, ShahDC, Lambert H, Leo G, Aeby N, et al. Novel contact force sensor incorporated in irrigated radiofrequency ablation catheter predicts lesion size and incidence of steam pop and thrombus. Circ Arrhythm Electrophysiol. 2008;1(5):354-362.

4. Calzolari V, De Mattia L, Indiani S, Crosato M, Furlanetto A, Licciardello C, et al. In vitro validation of the lesion size index to predict lesion width and depth after irrigated radiofrequency ablation in a porcine model. JACC Clin Electrophysiol. 2017;3(10):1126-1135.

5. Mattia L, Crosato M, Indiani S, Causin E, Licciardello C, Maria Squasi PA, et al. Prospective evaluation of lesion index-guided pulmonary vein isolation technique in patients with paroxysmal atrial fibrillation: 1-year follow-up. J Atr Fibrillation. 2018;10(6):1858.

6. Beinart R, Abbara S, Blum A, Ferencik M, Heist K, Ruskin J, et al. Left Atrial Wall Thickness Variability Measured by CT Scans in Patients Undergoing Pulmonary Vein Isolation. J Cardiovasc Electrophysiol, 2011;22:1232-1236.

7. Kirchhof P, Benussi S, Kotecha D, Ahlsson A, Atar D, Casadei B, et al. 2016 ESC Guidelines for the management of atrial fibrillation developed in collaboration with EACTS. Europace. 2016;18(11):16091678 .

8. Galeazzi M, Ficili S, Dottori S, Elian MA, Pasceri V, Venditti F, et al. Pain perception during esophageal warming due to radiofrequency catheter ablation in the left atrium. J Interv Card Electrophysiol. 2010;27(2):109-115.

9. Yamaguchi T, Fukui A, Node K. Bipolar Voltage Mapping for the Evaluation of Atrial Substrate: Can We Overcome the Challenge of Directionality? J Atr Fibrillation. 2019;11(5):2116.

10. Calkins H, Kuck KH, Cappato R, Brugada J, Camm AJ, Chen SA, et al. 2012 HRS/EHRA/ECAS Expert Consensus Statement on Catheter and Surgical Ablation of Atrial Fibrillation: recommendations for patient selection, procedural techniques, patient management and follow-up, definitions, endpoints, and research trial design. Europace. 2012;14(4):528-606.

11. Van Gelder IC, Healey JS, Crijns HJGM, Wang J, Hohnloser SH, Gold MR, et al. Duration of devicedetected subclinical atrial fibrillation and occurrence of stroke in ASSERT. Eur Heart J. 2017;38:13391344 .

12. Aryana A, Heist EK, D'Avila A, Holmvang G, Chevalier J, Ruskin JN, et al. Pain and anatomical locations of radiofrequency ablation as predictors of esophageal temperature rise during pulmonary vein isolation. J Cardiovasc Electrophysiol. 2008;19(1):32-38.

13. Mansour M, Ruskin J, Keane D. Efficacy and safety of segmental ostial versus circumferential extraostial pulmonary vein isolation for atrial fibrillation. J Cardiovasc Electrophysiol. 2004;15(5):532-537.

14. Nilsson B, Chen X, Pehrson S, Svendsen JH. The effectiveness of a high output/short duration radiofrequency current application technique in segmental pulmonary vein isolation for atrial fibrillation. Europace. 2006;8(11):962-965.

15. Kanj MH, Wazni O, Fahmy T, That S, Patel D, Elayi C, et al. Pulmonary vein antral isolation using an open irrigation ablation catheter for the treatment of atrial fibrillation. J Am Coll Cardiol. 2007;49:1634-1641.

16. Winkle RA, Mead RH, Engel G, Patrawala RA. Atrial fibrillation ablation: "perpetual motion" of open irrigated tip catheters at $50 \mathrm{~W}$ is safe and improves outcomes. Pacing Clin Electrophysiol. 2011;34(5):531-539.

17. Chen S, Schmidt B, Bordignon S, Urbanek L, Tohoku S, Bologna F, et al. Ablation Index-guided $50 \mathrm{~W}$ ablation for pulmonary vein isolation in patients with atrial fibrillation: Procedural data, lesion analysis, and initial results from the FAFA AI High Power Study. J Cardiovasc Electrophysiol. 2019;30(12):2724-2731.

18. Pambrun T, Durand C, Constantin M, et al. High-Power (40-50 W) Radiofrequency Ablation Guided by Unipolar Signal Modification for Pulmonary Vein Isolation: Experimental Findings and Clinical Results. Circ Arrhythm Electrophysiol. 2019;12(6):e007304.doi:10.1161/CIRCEP.119.007304. 
19. Barkagan M, Contreras-Valdes FM, Leshem E, Buxton AE, Nakagawa H, Anter E. High-power and short-duration ablation for pulmonary vein isolation: Safety, efficacy and long-term durability. J Cardiovasc Electrophysiol. 2018;29(9):1287-1296.doi:10.1111/jce.13651.

20. Pappone C, Manguso F, Vicedomini G, Gugliotta F, Santinelli O, Ferro A, et al. Prevention of iatrogenic atrial tachycardia after ablation of atrial fibrillation: a prospective randomized study comparing circumferential pulmonary vein ablation with a modified approach. Circulation. $2004 ; 110(19): 3036-$ 3042.

21. Winkle RA, Mohanty S, Patrawala RA, Mead RH, Kong MH, Engel G, Salcedo J, Trivedi CG, Gianni C, Jais P, Natale A, Day JD. Low complication rates using high power (45-50 W) for short duration for atrial fibrillation ablations. Heart Rhythm. 2019;16(2):165-169.

22. Bunch TJ, Day JD. Novel ablative approach for atrial fibrillation to decrease risk of esophageal injury. Heart Rhythm. 2008;5(4):624-627. doi:10.1016/j.hrthm.2007.11.00.

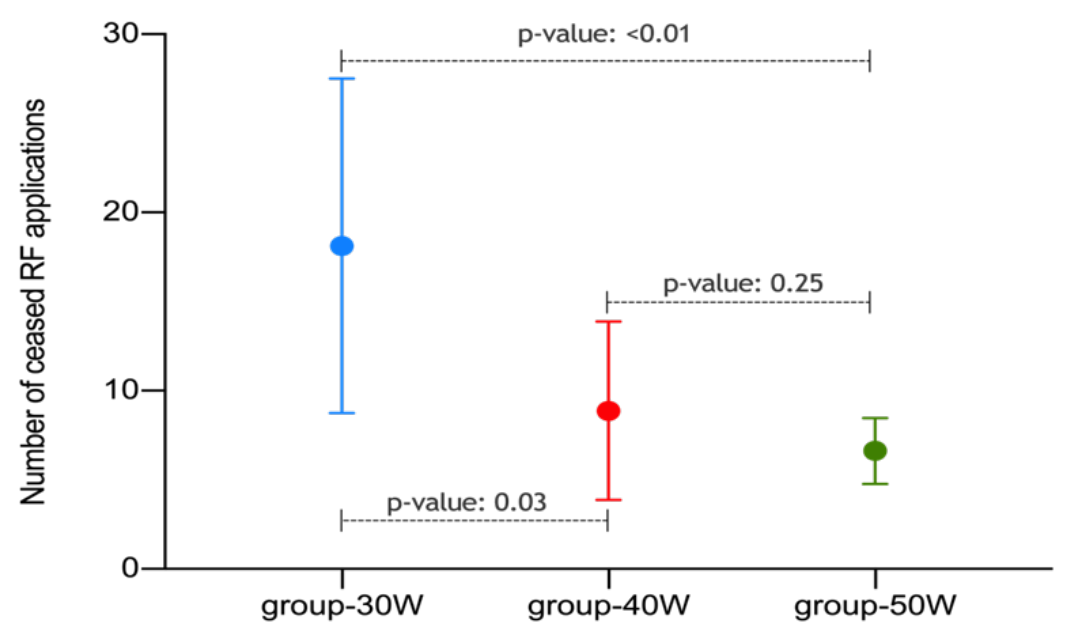

Figure1.Numberofceasedradiofrequencyapplicationsduetopaindepictedasmeanandstandarddeviationsinthestudiedgroups.

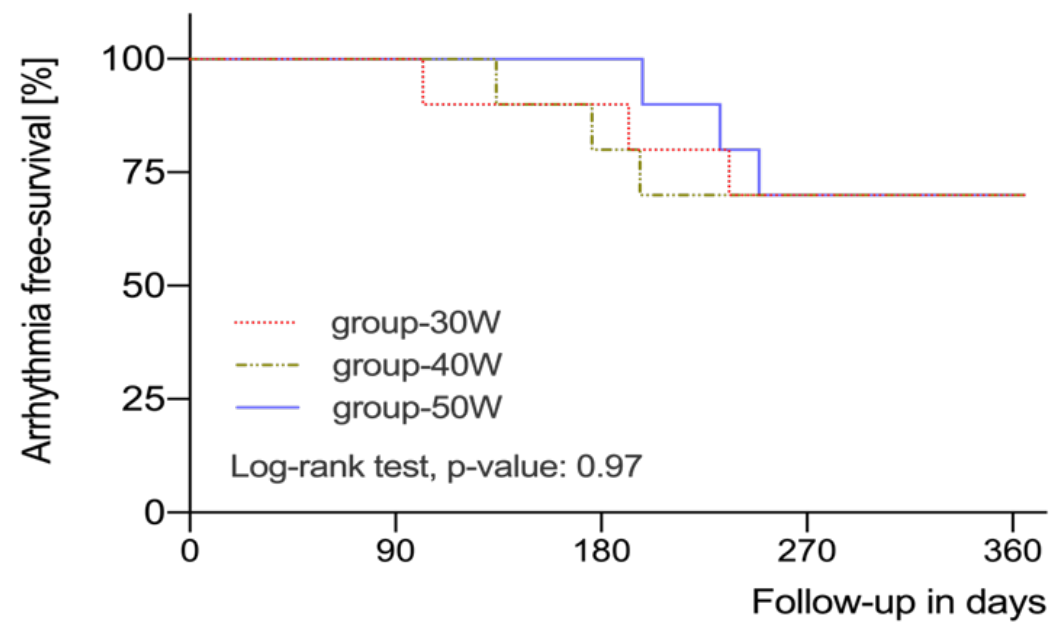

Figure 2. Kaplan-Meier estimates of freedom from any documented arrhythmia $>30$ seconds after a single procedure, without antiarrhythmic medications. 


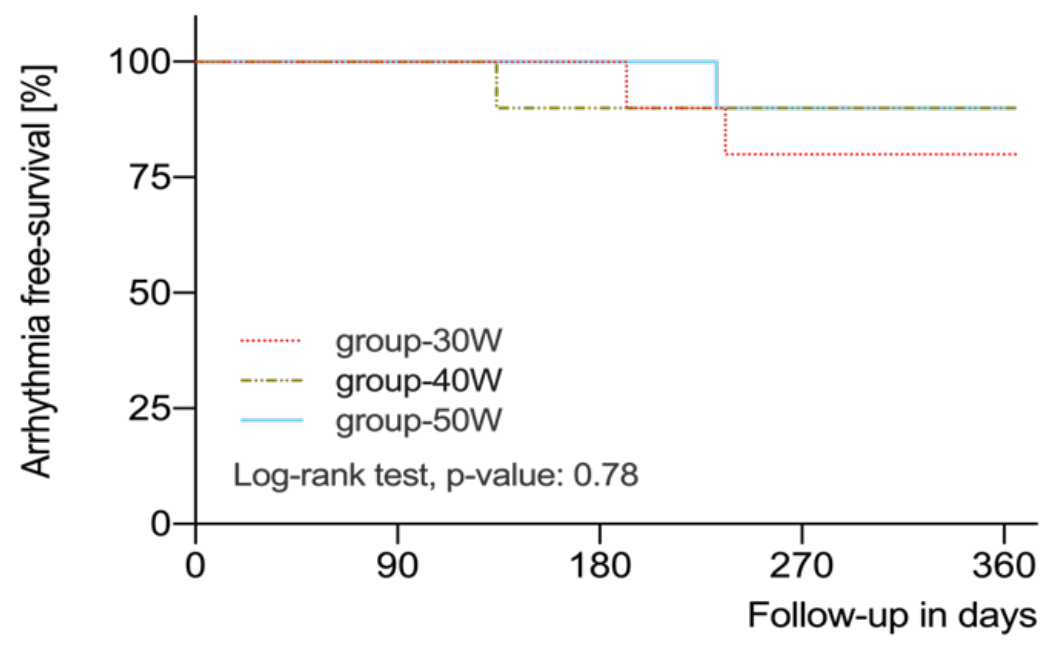

Figure 3. Kaplan-Meier estimates of freedom from any documented arrhythmia $>24$ hours after a single procedure, without antiarrhythmic medications.

Table 1. Comparison of patients characteristics between the studied groups: group-30W, group-40W and group-50W; BMI

Patients, $\mathrm{n}=30$

Age [years], mean (SD)

BMI $\left[\mathrm{kg} / \mathrm{m}^{2}\right]$, mean $(\mathrm{SD})$

BSA $\left[\mathrm{m}^{2}\right]$, mean $(\mathrm{SD})$

Sex - Male, in numbers (\%)

Paroxysmal atrial fibrillation, in numbers (\%)

Persistent atrial fibrillation, in numbers (\%)

CHA2DS2-VASc score, median (25-75\%)

Left atrium size $[\mathrm{mm}]$, mean (SD)

Left Ventricular Ejection Fracion [\%], mean (SD)

Left atrial appendage Vmax $[\mathrm{cm} / \mathrm{s}]$, mean (SD)

Table 2. Procedural characteristics.

Procedure

Procedure time [minutes], mean (SD)

Left atrial dwell time [minutes], mean (SD)

Voltage mapping time of left atrium [minutes], mean (SD)

Procedure time without Voltage mapping time [minutes], mean (SD)

Total RF time [minutes], mean (SD)

Total number of RF applications, mean (SD)

PVI time [minutes], mean (SD)

PVI-RF time [minutes], mean (SD)

PVI-Number of RF applications, mean (SD)

PVI-Time of a single RF application [seconds], mean (SD)

Contact force [gram], mean (SD)

Table 2. Procedural characteristics. Table 2. Proc

$1(\mathrm{n}=10)$

group-30W

$190(32)$

$154(53)$

$18(11)$

$169(28)$

$62(17)$

$133(43)$

$81(14)$

$51(7)$

$109(21)$

28(3)

15(2) $2(\mathrm{n}=10)$

group-40W

161(41)

113(29)

16(8)

$145(35)$

$38(10)$

134(47)

62(19)

$34(7)$

111(24)

18(1)

15(3) 
Table 2. Procedural characteristics.

Number of stopped RF applications due to pain, mean (SD)

Fluoroscopy time [minutes], mean (SD)

Fluoroscopy dose area product $\left[\mathrm{cGy} \cdot \mathrm{m}^{2}\right]$, mean $(\mathrm{SD})$
Table 2. Procedural characteristics.

$18(9)$

$6(3)$

$3377(2410)$
Table 2. Proc

$9(5)$

$6(2)$

2994(1048) 